Artigo Original

Original Article

Andreia Martins de Souza Cardoso

Mônica Marins da Silva ${ }^{1}$

Mônica Medeiros de Britto Pereira ${ }^{\top}$

\section{Consciência fonológica e a memória de trabalho de crianças com e sem dificuldades na alfabetização}

\author{
Phonological awareness and the working memory of \\ children with and without literacy difficulties
}

Descritores

Linguagem infantil Memória de curto prazo Alfabetização em saúde Aprendizagem Cognição

Keywords

Child language Memory, short-term Health literacy Learning Cognition

Endereço para correspondência: Andréia Martins de Souza Cardoso R. João de Oliveira Soares, 400, Jardim Camburi, Vitória (ES), Brasil, CEP: 29090-390.

E-mail: andreiasouz@yahoo.com.br

Recebido em: 26/12/2011

Aceito em: 29/1/2013

\section{RESUMO}

Objetivo: Investigar as habilidades de consciência fonológica e memória de trabalho, bem como a sua influência no processo de alfabetização em um grupo de crianças intelectualmente normais. Métodos: Participaram desta pesquisa 40 crianças de 7 anos e 6 meses a 8 anos, intelectualmente normais, dos segundo e terceiro anos do ensino fundamental. Estas foram organizadas em dois grupos com 20 cada, sendo um com dificuldade na alfabetização, e outro sem alterações nesse processo. Esses participantes foram submetidos ao teste RAVEN do quociente de inteligência, à avaliação audiométrica, ao teste de Consciência Fonológica - Instrumento de Avaliação Sequencial, à prova escrita de ditado e ao teste de memória de trabalho. Resultados: Os indivíduos que se encontram na fase alfabética apresentaram bom desenvolvimento da consciência fonológica e $85 \%$ deles, elevado desempenho da memória de trabalho. As crianças na fase silábico-alfabética apresentaram alterações na consciência fonológica e 91,6\% delas mostraram um mediano desempenho da memória de trabalho. Os sujeitos que se encontram nas fases silábica e pré-silábica tiveram mais dificuldades na consciência fonológica do que aqueles na silábico-alfabética, e obtiveram um baixo desempenho da memória de trabalho. Houve diferença entre as médias dos grupos para os testes CONFIAS e memória de trabalho $(\mathrm{p}<0,0001)$. Houve uma correlação também significativa, $r=0,78$, com $\mathrm{p}=0,01$, entre as habilidades de consciência fonológica e memória de trabalho para o total de sujeitos da amostra. Conclusões: Verificou-se que, na medida em que os níveis de consciência fonológica e memória de trabalho se elevam, a fase de alfabetização da criança também avança, sendo, portanto, medidas diretamente proporcionais.

\begin{abstract}
Purpose: To investigate phonological awareness and working memory skills as well as their influence on the literacy process in a group of intellectually normal children. Methods: Forty intellectually normal children (7.6-8.0 years) from the second and third grades of elementary school participated. Children were organized in two groups (20 children each): one with and another without literacy difficulties. These participants underwent RAVEN's intelligence quotient test, audiometric assessment, CONFIAS test of phonological awareness, written spelling task, and working memory test. Results: Children in the alphabetic phase presented a good development of phonological awareness, and $85 \%$ of them showed a high-performance working memory. Children in the syllabic-alphabetic phase had changes in phonological awareness, and $91.6 \%$ of them showed an average working memory performance. The subjects at pre-syllabic and syllabic phases demonstrated more difficulties in phonological awareness than those at syllabic-alphabetic and had a poor working memory performance. Between-group differences were observed for CONFIAS and working memory tests $(\mathrm{p}<0.0001)$. There was also a significant correlation $(\mathrm{r}=0.78$, $\mathrm{p}=0.01$ ) between the skills of phonological awareness and working memory for the total sample of individuals. Conclusions: Based on these results, it was found that as phonological awareness and working memory levels increased, the literacy phase also advanced, therefore showing that these are directly proportional measures.
\end{abstract}

Trabalho realizado na Universidade Veiga de Almeida - UVA - Rio de Janeiro (RJ), Brasil.

(1) Programa de Pós-graduação (Mestrado) em Fonoaudiologia, Universidade Veiga de Almeida - UVA - Rio de Janeiro (RJ), Brasil.

Conflito de interesse: nada a declarar. 


\section{INTRODUÇÃO}

O código escrito é uma forma de representação linguística que implica na habilidade de compreender ideias, memorizar informações e transmitir mensagens, possibilitando ao indivíduo a interação com o mundo letrado no qual está inserido ${ }^{(1-3)}$. Durante o processo de aprendizagem escolar, muitas crianças apresentam dificuldade na alfabetização por diversas causas. A memória é um dos aspectos mais importantes que possibilita a aprendizagem. Alterações nela podem ocasionar uma dificuldade no armazenamento das informações ensinadas, dificultando, portanto, a aquisição da leitura e da escrita ${ }^{(4,5)}$.

O processo do aprendizado da leitura e da escrita requer esforço e um ambiente estimulante ${ }^{(6,7)}$. Ser alfabetizado significa adquirir a habilidade de codificar a língua oral em escrita e decodificar a escrita em oral. Ler e escrever também se referem à apreensão e compreensão de significados expressos em língua escrita (ler) e expressão dos mesmos por meio da língua escrita (escrever) ${ }^{(6,8,9)}$.

Os processamentos visoespacial e fonológico são importantes para a aquisição da linguagem escrita. As informações visuais e fonológicas, uma vez percebidas, são armazenadas na memória de trabalho e posteriormente transferidas para a de longo prazo, possibilitando o aprendizado da associação fonema-grafema ${ }^{(1,10,11)}$.

Por meio da memória é possível realizar o armazenamento e a recuperação das informações linguística, oral ou escrita. Uma disfunção nesses processos pode afetar a habilidade de leitura e escrita ${ }^{(12,13)}$.

Durante o processo de alfabetização, as informações fonológicas e visuais precisam ser registradas na memória de trabalho e devem ser transferidas para a de longo prazo a fim de ocasionar o aprendizado do idioma escrito ${ }^{(14,15)}$. Devido a essa importância da memória de trabalho no processo de aprendizagem alfabética, tornam-se indispensáveis as avaliações da consciência fonológica e da memória de trabalho em crianças que se encontram na alfabetização.

Nesse sentido, o objetivo do presente estudo foi investigar as habilidades de consciência fonológica e de memória de trabalho, bem como a sua influência no momento de aprendizagem da alfabetização em um grupo de crianças intelectualmente normais com e sem dificuldades.

A integridade da memória de trabalho, além de outros aspectos, permite o processo de compreender a leitura e a escrita, possibilitando a formação de um cidadão funcionalmente letrado, ou seja, um sujeito capaz de fazer uso da linguagem escrita para sua necessidade individual ${ }^{(1,2,4,10)}$.

\section{MÉTODOS}

Esta pesquisa foi iniciada após a aprovação do Comitê de Ética em Pesquisa da Universidade Veiga de Almeida, Rio de Janeiro, sob número de resolução 274/11. Todos os responsáveis envolvidos na pesquisa assinaram o termo de consentimento livre e esclarecido.

Participaram desta pesquisa 40 crianças de 7 anos e 6 meses a 8 anos, intelectualmente normais, que cursavam os segundo e terceiro anos do ensino fundamental organizadas em dois grupos de 20 crianças. O primeiro deles incluía crianças com dificuldade na alfabetização (Grupo 1) e o outro era composto por aquelas sem alterações nesse processo (Grupo 2), como pode ser visto na Tabela 1.
Tabela 1. Distribuição dos participantes em relação à idade e ao gênero

\begin{tabular}{lcccc}
\hline Número de participantes & $\mathrm{n}$ & $\begin{array}{c}\text { Média de } \\
\text { idades }\end{array}$ & $\begin{array}{c}\text { Idade } \\
\text { mínima }\end{array}$ & $\begin{array}{c}\text { Idade } \\
\text { máxima }\end{array}$ \\
\hline Grupo 1 (gênero masculino) & 14 & 7,7 & 7,6 & 8,0 \\
Grupo 1 (gênero feminino) & 6 & 7,7 & 7,6 & 8,0 \\
Grupo 2 (gênero masculino) & 6 & 7,7 & 7,6 & 8,0 \\
Grupo 2 (gênero feminino) & 14 & 7,7 & 7,6 & 8,0 \\
\hline
\end{tabular}

Fizeram parte do Grupo 114 crianças do gênero masculino e 6 do feminino. Já no Grupo 2 havia 14 do feminino e 6 do masculino, sem dificuldades de alfabetização. A média de idades e as idades mínima e máxima foram semelhantes para todos os participantes da pesquisa.

Estima-se que os sujeitos apresentassem o mesmo nível socioeconômico, pois eram de escolas públicas do mesmo município. Foram excluídas crianças com alterações no exame audiométrico e no teste do quociente de inteligência (QI). Também foram excluídos os participantes que apresentavam atraso no ingresso escolar, repetência escolar, atraso de linguagem, alterações neurológicas e visuais, segundo informações fornecidas pelos pais na entrevista inicial.

Para a realização deste estudo, foram utilizados os seguintes instrumentos: teste RAVEN de QI - matrizes progressivas coloridas $^{(16)}$; avaliação audiométrica - audiometria tonal limiar; CONFIAS consciência fonológica ${ }^{(17)}$, considerando tarefas de consciências silábica e fonêmica ${ }^{(17)}$; prova escrita de ditado de Capovilla e Capovilla ${ }^{(18)}$ e teste de memória de trabalho, elaborado por $\mathrm{Curi}^{(1)}$.

As crianças foram encaminhadas pelos professores das escolas municipais à realização do teste de QI, que foi aplicado pela psicóloga de uma Unidade de Saúde do município. Posteriormente, os alunos passaram pela avaliação audiométrica. Todas aquelas que apresentaram alterações nesses testes foram excluídas do estudo e encaminhadas para acompanhamento médico e fonoaudiológico. Com isso, foi possível selecionar uma amostra de 40 indivíduos cognitivamente normais e com limiares auditivos dentro dos padrões de normalidade. Em seguida, foram aplicadas as avaliações fonoaudiológicas. A prova escrita de ditado foi a primeira a ser realizada a fim de identificar o nível de alfabetização. Desse modo, dois grupos foram constituídos, sendo um com 20 crianças em atraso no processo de alfabetização, ou seja, nas fases pré-silábica, silábica ou silábico-alfabética (Grupo 1), e outro com 20 alunos com escrita na fase alfabética (Grupo 2). O CONFIAS foi utilizado em seguida para avaliar a consciência fonológica. Posteriormente, foi realizado pela psicóloga em todos os participantes da pesquisa o teste da memória de trabalho. Após as avaliações, os resultados foram submetidos à análise estatística, com testes para estimar a correlação e as diferenças entre a consciência fonológica e a memória de trabalho de cada grupo (testes não paramétrico de correlação de Spearman, $t$ e não paramétricos pareados de Wilcoxon e Kruskal-Wallis).

\section{RESULTADOS}

A Tabela 2 mostra as médias, os desvios padrão e os valores mínimos e máximos dos testes de sílabas, fonemas, total CONFIAS e memória de trabalho para os Grupos 1 e 2. 
Tabela 2. Estatística descritiva dos Grupos 1 e 2

\begin{tabular}{|c|c|c|c|c|c|c|c|c|}
\hline \multirow{2}{*}{$\begin{array}{l}\text { Crianças com atraso na } \\
\text { alfabetização }\end{array}$} & \multicolumn{4}{|c|}{ Grupo 1 - com atraso na alfabetização (20) } & \multicolumn{4}{|c|}{ Grupo 2 - sem atraso na alfabetização (20) } \\
\hline & Mínimo & Máximo & Média & $\begin{array}{l}\text { Desvio } \\
\text { padrão }\end{array}$ & Mínimo & Máximo & Média & $\begin{array}{l}\text { Desvio } \\
\text { padrão }\end{array}$ \\
\hline Teste de sílabas & 11 & 32 & 22,1 & 5,2 & 33 & 40 & 37,8 & 1,8 \\
\hline Teste de fonemas & 5 & 20 & 10,7 & 3,9 & 18 & 29 & 26,3 & 2,9 \\
\hline Total CONFIAS & 16 & 44 & 32,8 & 7,8 & 51 & 69 & 64,1 & 4,4 \\
\hline Teste de MT & 16 & 27 & 22,3 & 3,6 & 24 & 36 & 31,3 & 3,2 \\
\hline
\end{tabular}

Legenda: MT = memória de trabalho; CONFIAS = consciência fonológica instrumento de avaliação sequêncial

A Tabela 3 evidencia a diferença entre as médias e os níveis de significância das variáveis testadas dos grupos com e sem dificuldades na alfabetização.

Com relação ao teste de consciência fonológica (CONFIAS), a média dos resultados do grupo de crianças com dificuldades na alfabetização foi mais baixa do que aquelas sem tal problema (32,8 e 64,1, respectivamente). O desvio padrão do Grupo 1 foi 7,8 e do 2, 4,4. Esses números reforçam elementos para se afirmar que os grupos eram suficientemente distintos para a variável de habilidades fonológicas. No grupo dos 20 indivíduos no estágio alfabético, todos apresentaram escores acima de 46 no CONFIAS, determinando um bom desenvolvimento da consciência fonológica. Os outros 20 participantes, das fases pré-silábica, silábica e silábico-alfabética, apresentaram escores abaixo de 46 no CONFIAS, apresentando uma dificuldade na habilidade de consciência fonológica.

No teste da memória de trabalho, a média dos resultados do grupo de crianças com dificuldade na alfabetização foi de 22,3 , significativamente mais baixa do que a do outro $(31,3)$. O desvio padrão do Grupo 1 foi 3,6 e o do 2, 3,2. Os resultados do teste de memória de trabalho mostram uma diferença entre as médias dos grupos ( 9 pontos, $\mathrm{p}<0,001$ ). Esta evidencia que, em relação ao desempenho da memória de trabalho, tratavamse de grupos diferentes. A maioria dos indivíduos em fase alfabética apresentou um elevado desempenho da memória de trabalho. Dos 20 alfabéticos, $85 \%$ apresentaram alto desempenho de memória de trabalho, e 15\%, um mediano. Já o grupo de crianças com atraso no processo de alfabetização teve um resultado mais heterogêneo. Os indivíduos das fases pré-silábica e silábica mostraram um desempenho inferior da memória de trabalho. Naquelas incluídas na silábico-alfabética, 91,6\% delas apresentaram uma performance mediana da memória de trabalho, e $8,3 \%$, baixa.

\section{Resultados das correlações e níveis de significância entre a consciência fonológica e a memória de trabalho}

As Tabelas 4 e 5 mostram os resultados do teste de Spearman. A partir delas é possível observar as correlações entre as variáveis (sílabas, fonemas, memória de trabalho e CONFIAS) e seus respectivos níveis de significância para os Grupos 1 e 2 (Tabela 4) separadamente e os dois juntos (Tabela 5). Representações visuais desses dados podem ser encontradas nas Figuras 1 (Grupo 1), 2 (Grupo 2) e 3 (Grupos 1 e 2 juntos). Como se pode observar na Tabela 4 , houve correlações positivas no Grupo 1 entre as quatro variáveis estudadas (sílabas, fonemas, memória de trabalho e CONFIAS); no Grupo 2 foram notadas entre sílabas, fonemas e CONFIAS. Vale ressaltar que a variável CONFIAS é composta pela soma dos escores da sílaba e do fonema no teste, mas não entre as mesmas com a memória de trabalho. Tal resultado é justificado pelo fato de que a maioria dos indivíduos do Grupo 2 apresentou um elevado desempenho da consciência fonológica e da memória de trabalho, o que é esperado para o grupo de crianças sem

Tabela 3. Diferença entre as médias dos grupos com e sem dificuldade na alfabetização

\begin{tabular}{lccc}
\hline $\begin{array}{l}\text { Diferença absoluta entre as médias de } \\
\text { cada teste realizado }\end{array}$ & $\begin{array}{c}\text { Diferença absoluta } \\
\text { entre as médias }\end{array}$ & Valor de $\mathrm{p}$ & $\begin{array}{c}\text { Diferença percentual } \\
\text { entre as médias }\end{array}$ \\
\hline Teste de sílabas & 15,7 & $<0,001$ & 70,8 \\
Teste de fonemas & 15,7 & $<0,001$ & 146,9 \\
Teste de MT & 9,0 & $<0,001$ & 40,4 \\
Total CONFIAS & 31,3 & $<0,001$ & 95,6 \\
\hline
\end{tabular}

Teste $t(\mathbf{p}<0,05)$

Legenda: MT = memória de trabalho; CONFIAS = consciência fonológica instrumento de avaliação sequêncial

Tabela 4. Resultados das correlações entre os testes CONFIAS e memória de trabalho dos Grupos 1 e 2

\begin{tabular}{lcccccccc}
\hline Correlações entre os testes & \multicolumn{4}{c}{ Grupo 1 - com atraso na alfabetização (20) } & \multicolumn{4}{c}{ Grupo 2 sem atraso na alfabetização (20) } \\
\cline { 2 - 8 } das crianças do Grupo 1 & Sílabas & Fonemas & MT & CONFIAS & Sílabas & Fonemas & MT & CONFIAS \\
\hline Sílabas & - & $0,56^{*}$ & $0,77^{* *}$ & $0,9^{\star *}$ & - & $0,48^{*}$ & $-0,09(0,7)$ & $0,8^{* *}$ \\
Fonemas & - & - & $0,55^{*}$ & $0,82^{\star *}$ & - & - & $-0,06(0,8)$ & $0,88^{\star *}$ \\
MT & - & - & - & $0,68^{\star *}$ & - & - & - & $-0,15(0,5)$ \\
\hline
\end{tabular}

* Correlação no nível de 0,05 (bicaudal); ** correlação no nível de 0,01 (bicaudal)

Legenda: MT = memória de trabalho; CONFIAS = consciência fonológica instrumento de avaliação sequêncial 
Tabela 5. Resultados das correlações entre os testes CONFIAS e memória de trabalho da amostra total

\begin{tabular}{lcccc}
\hline Correlações entre os & \multicolumn{4}{c}{ Amostra total de 40 crianças } \\
\cline { 2 - 5 } testes da amostra total & Sílabas & Fonemas & MT & CONFIAS \\
\hline Sílabas & - & $0,88^{*}$ & $0,8^{*}$ & $0,96^{*}$ \\
Fonemas & - & - & $0,78^{*}$ & $0,96^{*}$ \\
MT & - & - & - & $0,78^{*}$ \\
\hline${ }^{*}$ Correlação no nível de 0,01 (bicaudal); as estatísticas descritivas e o teste de \\
Spearman foram rodados no software SPSS 18.0 \\
Legenda: MT = memória de trabalho; CONFIAS = consciência fonológica instru- \\
mento de avaliação sequêncial
\end{tabular}

dificuldade na alfabetização. Para os dois grupos juntos houve correlações positivas para as quatro características pesquisadas.

\section{Resultado dos testes de diferenças entre os grupos}

Os testes de Wilcoxon e Kruskal-Wallis também foram realizados para confirmar a diferença entre os Grupos 1 e 2. Seus resultados confirmam a hipótese de que os grupos com e sem atraso na alfabetização são diferentes para todas as variáveis, mostrando um nível de significância de 0,01.

\section{DISCUSSÃO}

O presente estudo mostrou que a maioria das crianças no estágio alfabético de escrita obteve um bom desenvolvimento da consciência fonológica e grande parte dos alunos que não apresentam esta hipótese de escrita teve dificuldades. Tais conclusões estão de acordo com algumas pesquisas realizadas sobre consciência fonológica ${ }^{(6-8,17,19-22)}$, que confirmam a existência de correlação entre os níveis de consciência fonológica e de aquisição da linguagem escrita.

Vários estudos expõem opiniões diferentes de autores sobre a relação entre a consciência fonológica e a memória de trabalho. Alguns autores ${ }^{(1,3,13)}$ afirmam que essa relação é positiva, e outros ${ }^{(4)}$ que é negativa, mas a maioria defende a ideia de que a memória de trabalho e a consciência fonológica fazem parte do processamento fonológico. Portanto, existe correlação entre ambos, evidenciando uma relação de dependência entre essas duas habilidades, uma vez que a natureza da informação processada é fonológica. As operações de memória, representadas pela codificação, pelo armazenamento e pela recuperação da informação, são necessárias para a execução de tarefas de consciência fonológica.

Essa correlação é evidenciada na medida em que grande parte das crianças que apresentaram dificuldades na consciência fonológica e no teste de memória obteve escores que caracterizavam baixo ou mediano desempenho da memória de trabalho. A maioria dos participantes com bom desenvolvimento da consciência fonológica apresentou um alto da memória de trabalho. Dessa forma, à medida que as habilidades de consciência fonológica aumentaram, o nível de desempenho da memória de trabalho também o fez.

A partir da hipótese de correlação entre a consciência fonológica e a memória de trabalho, alguns autores buscaram verificar a influência de ambas no aprendizado da linguagem escrita. Tais pesquisadores declaram que a memória de

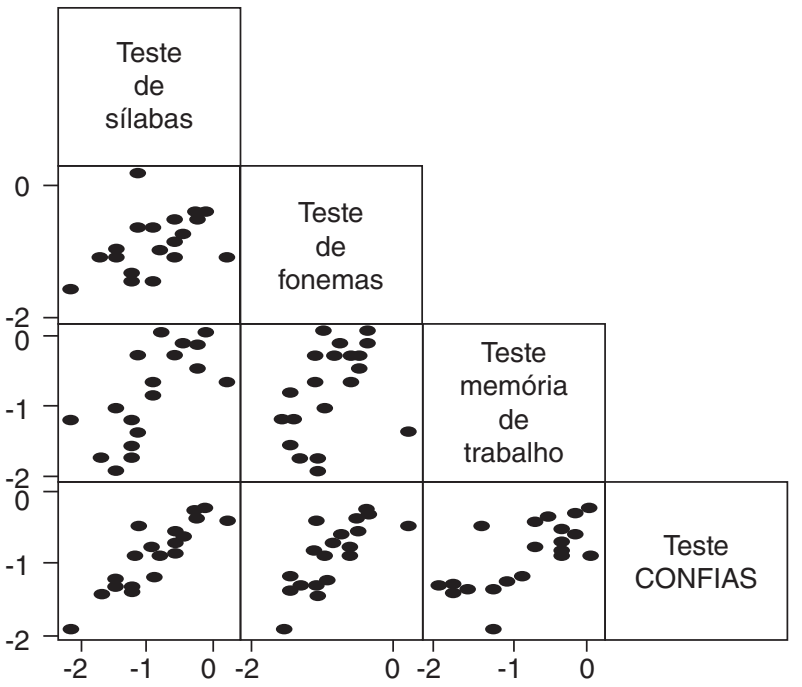

Figura 1. Matriz de dispersão entre os testes de sílabas, fonemas, memória de trabalho e CONFIAS (grupo de alunos com atraso na alfabetização)

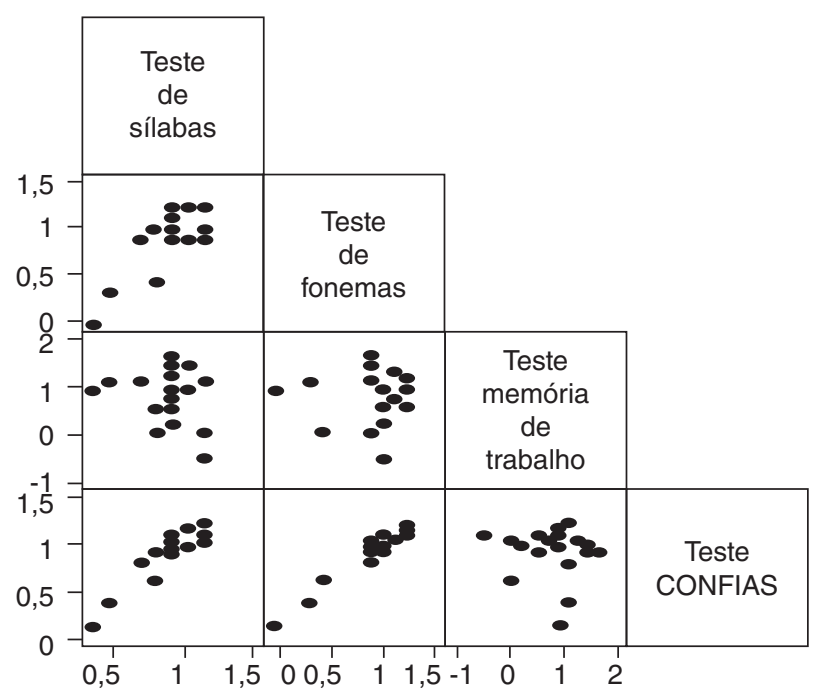

Figura 2. Matriz de dispersão entre os testes de sílabas, fonemas, memória de trabalho e CONFIAS (grupo de alunos sem atraso na alfabetização)

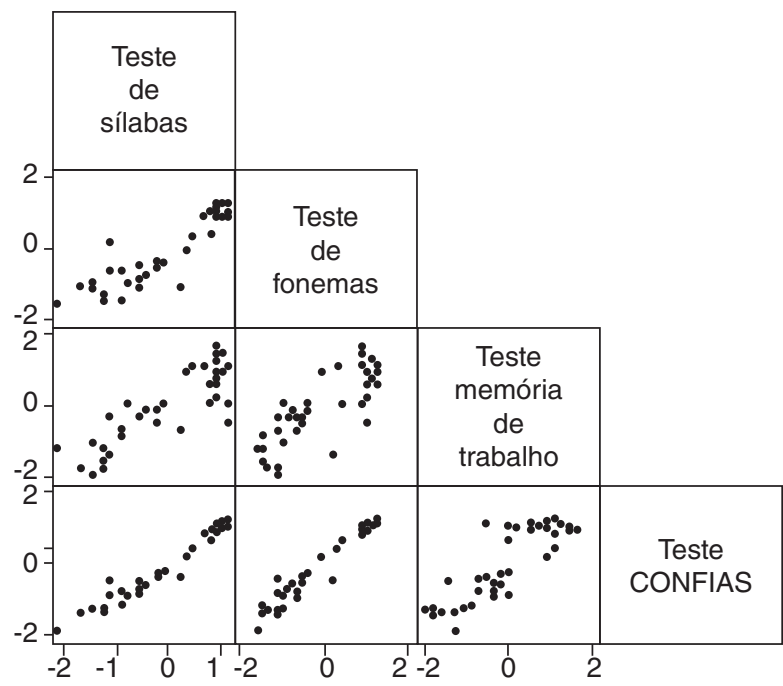

Figura 3. Matriz de dispersão entre os testes de sílabas, fonemas, memória de trabalho e CONFIAS (amostra total de 40 indivíduos) 
trabalho mantém as informações fonológicas e de símbolos da linguagem escrita temporariamente armazenadas e ativas, possibilitando sua transferência para a de longo prazo, resultando no aprendizado ${ }^{(2,10,14)}$.

Os resultados deste artigo mostraram que os participantes que no nível de escrita alfabético apresentaram um bom desenvolvimento da consciência fonológica, e a maioria deles, um elevado desempenho da memória. As crianças na fase silábico-alfabética tiveram alterações na consciência fonológica, e a maioria delas obteve um desempenho de memória mediano. Já a maioria dos sujeitos nas fases silábica e pré-silábica mostrou mais dificuldades nas habilidades de consciência fonológica do que aqueles na silábico-alfabética, e obteve um baixo desempenho de memória. Sendo assim, esses resultados estão de acordo com os estudos citados, uma vez que mostraram a influência das alterações na consciência fonológica e na memória de trabalho no processo de alfabetização de crianças. Porém, este estudo completa os demais por demonstrar que crianças intelectualmente normais podem ter mudanças na consciência fonológica e na memória de trabalho, determinando uma dificuldade no processo de alfabetização.

Estudos anteriores mostraram que a consciência fonológica e a memória de trabalho estão inter-relacionadas e são atividades cognitivas indissociáveis ${ }^{(10,11)}$. Do mesmo modo, o presente estudo reforça tais conclusões, mostrando que tais habilidades são desenvolvidas concomitantemente, e, nesse processo, atuam influenciando no processo de alfabetização. À medida que a consciência fonológica vai se desenvolvendo, o nível do desempenho da memória de trabalho também aumenta e vice-versa. No entanto, isso não significa que a consciência fonológica determina o desenvolvimento da memória de trabalho ou vice-versa. Somente é possível afirmar que quanto maiores forem os níveis de consciência fonológica e memória de trabalho, melhor é a fase de alfabetização da criança, sendo, portanto, medidas diretamente proporcionais. Dessa forma, pode-se dizer que o nível de desenvolvimento da consciência fonológica ocorre em conjunto com aquela da memória de trabalho, colaborando para determinar a fase de alfabetização de um indivíduo.

\section{CONCLUSÕES}

Por meio deste estudo foi possível verificar a existência de correlação entre consciência fonológica e memória de trabalho, bem como que alterações na consciência fonológica e na memória de trabalho podem influenciar o processo de alfabetização de crianças intelectualmente normais.

As habilidades de consciência fonológica se desenvolvem em conjunto com a memória de trabalho e à medida que a consciência fonológica vai se desenvolvendo o nível do desempenho de memória também aumenta e vice-versa. Quanto maiores forem os níveis de consciência fonológica e memória de trabalho, mais avançada é a fase de alfabetização da criança, sendo, portanto, medidas diretamente proporcionais. Os dados deste estudo apontam que a avaliação do desempenho de crianças em relação à consciência fonológica e à memória de trabalho, antes de se iniciar o processo de alfabetização, pode facilitar o prognóstico do processo de aquisição da linguagem escrita.

* AMSC foi responsável pela execução do projeto, elaboração do manuscrito, coleta e tabulação dos dados; MMBP foi responsável pela orientação geral, supervisão da coleta dos dados e das etapas de execução; MMS colaborou na orientação do projeto e em suas etapas e execução.

\section{REFERÊNCIAS}

1. Curi N. Atenção, memória e dificuldades de aprendizagem [tese] Campinas: Universidade Estadual de Campinas, Faculdade de Educação; 2002.

2. Rodrigues C. Contribuições da memória de trabalho para o processamento da linguagem. Evidências experimentais e clínicas [pós-doutorado] Santa Catarina: Universidade Federal de Santa Catarina, Programa de Pós-Graduação em Linguística, Letras e Artes; 2001.

3. Segers E, Verhoeven L. Long-term effects of computer training of phonological awareness in kindergarten. J Comput Assist Learn. 2005;21(1): 17-27

4. Gillam RB, Van Kleeck A. Phonological awareness training and shortterm working memory: clinical implications. Topics Lang Disord. 1996;17(1):72-81

5. Jeronymo RR, Galera A. A relação entre a memória fonológica e a habilidade lingüística de crianças de 4 a 9 anos. Pro Fono. 2000;12(12):55-60.

6. Maluf MR, Barrera SD. Consciência fonológica e linguagem escrita em pré-escolares. Psicol Reflex Crit. 1997;10(1):125-45.

7. Moraes J, Kolinsky R, Alégria J, ScliarCabral L. Alphabetic literacy and psychological structure. Letras de Hoje. 1998;33(4):61-79.

8. Capellini SA, Ciasca SM. Avaliação da consciência fonológica em crianças com distúrbio específico de leitura e escrita e distúrbio de aprendizagem. T Desenv. 2000;8(48):17-23.

9. Marcuschi LA. Oralidade e letramento. In: Marcuschi LA. Da fala para a escrita: atividades de retextualização. São Paulo: Cortez; 2001. p. 15-43.

10. Gindri G, Kesk-Soares M, Mota HB. Memória de trabalho, consciência fonológica e hipótese de escrita. Pro Fono. 2007;19(3):313-22.

11. Santos RM, Siqueira M. Consciência fonológica e memória. Fono Atual. 2002;5(20):48-53.

12. Gathercole S, Baddeley A. Working memory and language. Hove: Lawrence Eribaum; 1993

13. Oakhill J, Kyler F. The relations between phonological: awareness and working memory. J Exp Child Psycol. 2000;75(2):152-64.

14. Linassi LZ, Keske-Soares M, Mota HB. Habilidades de memória de trabalho e o grau de severidade do desvio fonológico. Pro Fono. 2005;17(3):383-92.

15. Lobo FS, Acrani IO, Avila CR. Tipo de estímulo e memória de trabalho fonológica. Rev Cefac. 2008;10(4):461-70.

16. Raven JC. Matrizes progressivas coloridas de raven. São Paulo: Casa do Psicólogo; 1987.

17. Moojen S, Lamprecht R, Santos RM, Freitas GM, Brodacz R, Siqueira M, et al. CONFIAS Consciência Fonológica: Instrumento de Avaliação Seqüencial. São Paulo: Casa do Psicólogo; 2003.

18. Pinheiro AMV. Leitura e Escrita: uma abordagem cognitiva. Campinas: Psy II; 1994.

19. Santamaria VL, Leitão PB, Assencio-Ferreira VJ. A consciência fonológica no processo de alfabetização. Rev Cefac. 2004;6(3):237-41.

20. Bride-Chang C, Ho C. Developmental issues in Chinese children character acquisition. J Educ Psychol. 2000;92(1):50-5.

21. Cárnio MS, Santos D. Evolução da consciência fonológica em alunos de ensino fundamental. Pro Fono. 2005;17(2):195-200.

22. Capovilla AGS, Capovilla FC. Efeitos do treino de consciência fonológica em crianças com baixo nível sócio-econômico. Psicol Reflex Crit. 2000;13(1):7-24. 